\title{
High-Order Implicit Shock Tracking
}

\author{
Matthew J. Zahr \\ University of Notre Dame \\ Aerospace and Mechanical Engineering \\ Email: mzahr@nd.edu
}

\begin{abstract}
Shock tracking, as an alternative method to shock capturing, aims to generate a mesh such that element faces align with shock surfaces and other non-smooth features to perfectly represent them with the inter-element jumps in the solution basis, e.g., in the context of a finite volume or discontinuous Galerkin (DG) discretization. These methods have been shown to enable highorder approximation of high-speed flows and do not require extensive refinement in non-smooth regions because, once the non-smooth features are tracked by the mesh, the solution basis approximates the remaining smooth features.

In previous work $[1,2]$, we introduced an implicit shock tracking framework that re-casts the geometrically complex problem of generating a mesh that conforms to all discontinuity surfaces as a PDE-constrained optimization problem. The optimization problem seeks to determine the flow solution and nodal coordinates of the mesh that simultaneously minimize an error-based indicator function and satisfy the discrete flow equations. A DG discretization of the governing equations is used as the PDE constraint to equip the discretization with desirable properties: conservation, stability, and high-order approximation (both solution and geometry). By using high-order elements, curved meshes are obtained that track curved shock surfaces to high-order accuracy. The optimization problem is solved using a sequential quadratic programming method that simultaneously converges the mesh and DG solution, which is critical to avoid nonlinear stability issues that would come from computing a DG solution on an unconverged (non-aligned) mesh.

In this talk, we present a number of extensions to the implicit shock tracking method aimed at improving robustness for complex problems, including: (1) introduction of shock-preserving, arbitrary-order element collapses in arbitrary dimensions to preserve solution features; (2) incorporation of pseudo-transient continuation to improve solver robustness with respect to the initial guess; and (3) the use of vanishing-viscosity as part of the optimization solver to prevent severe mesh motion and distortion. We use the proposed framework to solve a number of relevant inviscid, steady and unsteady, transonic and supersonic, inert and reacting flows in two and three dimensions, and demonstrate the potential of the method to provide accurate approximations to these difficult problems with local features on extremely coarse, high-order meshes.
\end{abstract}

\section{REFERENCES}

[1] Matthew J. Zahr and Per-Olof Persson. An optimization-based approach for high-order accurate discretization of conservation laws with discontinuous solutions. Journal of Computational Physics, 365:105-134, July 2018.

[2] Matthew J. Zahr, Andrew Shi, and Per-Olof Persson. Implicit shock tracking using an optimization-based high-order discontinuous Galerkin method. Journal of Computational Physics, 410:109385, June 2020. 